University of Wollongong

Research Online

Faculty of Engineering and Information

Faculty of Engineering and Information

Sciences - Papers: Part B

Sciences

2018

Synthesis of highly-stretchable graphene - poly(glycerol sebacate) elastomeric nanocomposites piezoresistive sensors for human motion detection applications

Yi Yan

University of Wollongong, yy619@uowmail.edu.au

Michael Potts

University of Wollongong

Zhengyi Jiang

University of Wollongong, jiang@uow.edu.au

Vitor Sencadas

University of Wollongong, victors@uow.edu.au

Follow this and additional works at: https://ro.uow.edu.au/eispapers1

Part of the Engineering Commons, and the Science and Technology Studies Commons

Research Online is the open access institutional repository for the University of Wollongong. For further information contact the UOW Library: research-pubs@uow.edu.au 


\title{
Synthesis of highly-stretchable graphene - poly(glycerol sebacate) elastomeric nanocomposites piezoresistive sensors for human motion detection applications
}

\author{
Abstract \\ Strain sensors capable of monitoring human motion are highly desirable not only to monitor in real time \\ complex body parts movement but also to assess the patients' health condition. In this work, highly \\ stretchable elastomeric nanocomposite strain sensors were developed by synthesis of poly(glycerol \\ sebacate) (PGS) prepolymer, followed by incorporation of the graphene flakes ( $\mathrm{fG}$ ) during the curing \\ procedure of the PGS elastomeric matrix. The PGS had Young's modulus (E) of $69 \pm 6 \mathrm{kPa}$, while the \\ sample with $10 \mathrm{wt} \% \mathrm{fG}$ had a $\mathrm{E}$ of $501 \pm 28 \mathrm{kPa}$, which is as soft as human skin. Moreover, the \\ incorporation of the fG filler led to an increase of the matrix cross-linking density, from $8.0 \pm 0.6 \mathrm{~mol} / \mathrm{m} 3$, \\ up to $35.5 \pm 1.4 \mathrm{~mol} / \mathrm{m} 3$, recorded for the pure matrix and for the sample with $10 \mathrm{wt} \% \mathrm{fG}$, respectively. \\ The electromechanical behavior of the nanocomposite samples showed that the resistance changes \\ linearly with the applied strain, until a maximum strain of $50 \%$, and is independent of the stroke speed or \\ applied strain. The nanocomposite sensor sensibility was calculated through the gauge Factor (GF), and a \\ value of $\sim 2$ was obtained for all the processed strain gauges. Furthermore, the developed sensors \\ showed a remarkable capability to monitor the bending movement of the finger. Finally, the performance \\ revealed by the synthetized piezoresistive sensors will contribute to the next generation of highly \\ stretchable smart materials for sensing and control human motion with minimum discomfort. \\ Disciplines \\ Engineering | Science and Technology Studies

\section{Publication Details} \\ Yan, Y., Potts, M., Jiang, Z. \& Sencadas, V. (2018). Synthesis of highly-stretchable graphene - poly(glycerol \\ sebacate) elastomeric nanocomposites piezoresistive sensors for human motion detection applications. \\ Composites Science and Technology, 162 14-22.
}




\title{
Synthesis of highly-stretchable graphene - poly(glycerol sebacate) elastomeric nanocomposites piezoresistive sensors for human motion detection applications
}

\author{
Yi Yan $^{1+}$, Michael Potts ${ }^{1+}$, Zhengyi Jiang ${ }^{1, *}$, Vitor Sencadas ${ }^{1,2 *}$
}

${ }^{1}$ School of Mechanical, Materials and Mechatronic Engineering, University of Wollongong, Wollongong, NSW 2522, Australia

${ }^{2}$ ARC - Center of Excellence for Electromaterials Science, University of Wollongong, 2522

NSW, Australia

+ both authors contribute equally

*Corresponding author e-mail address: victors@uow.edu.au; jiang@uow.edu.au

\begin{abstract}
:
Strain sensors capable of monitoring human motion are highly desirable not only to monitor in real time complex body parts movement but also to assess the patients' health condition. In this work, highly stretchable elastomeric nanocomposite strain sensors were developed by synthesis of poly(glycerol sebacate) (PGS) prepolymer, followed by incorporation of the graphene flakes (fG) during the curing procedure of the PGS elastomeric matrix. The PGS had Young's modulus $(E)$ of $69 \pm 6 \mathrm{kPa}$, while the sample with $10 \mathrm{wt} \% \mathrm{fG}$ had a $E$ of $501 \pm 28 \mathrm{kPa}$, which is as soft as human skin. Moreover, the incorporation of the fG filler led to an increase of the matrix crosslinking density, from $8.0 \pm 0.6 \mathrm{~mol} / \mathrm{m}^{3}$, up to $35.5 \pm 1.4 \mathrm{~mol} / \mathrm{m}^{3}$, recorded for the pure matrix and for the sample with $10 \mathrm{wt} \% \mathrm{fG}$, respectively. The electromechanical behaviour of the nanocomposite samples showed that the resistance changes linearly with the applied strain, until a maximum strain of $50 \%$, and is independent of the stroke speed or applied strain. The nanocomposite sensor sensibility was calculated through the gauge Factor (GF), and a value of $\sim 2$ was obtained for all the processed strain gauges. Furthermore, the developed sensors showed a remarkable capability to monitor the bending movement of the finger. Finally, the performance revealed by the synthetized piezoresistive sensors will contribute to the next generation of highly stretchable smart materials for sensing and control human motion with minimum discomfort.
\end{abstract}




\section{Introduction}

Monitoring human motion is a difficult task due to the complex movements that the body can perform. These motions can fall in two categories: large-scale motions such the bending of the hands, fingers, and limbs, and the small-scale movements such the motion of the chest and necking during breathing swallowing, and speaking [1]. The sensors capable to accurately track these motions must have a good stretchability to follow the body parts submitted to large strains, and suitable sensibility to monitor small strain movements [2-4]. Strain sensors based in metal alloys or semiconductors (e.g. silicon-based) have a gauge factor (GF) between 0.5 and more than 100, however, their stretchability is limited to less than 5\% strain, and therefore they are incompatible with the human body motion $[4,5]$.

Recently, skin-mountable and wearable sensors have been proposed by using conductive nanomaterials, e.g. carbon nanotubes (CNT), silver and gold particles, or graphene, deposited on the surface or blended with polymeric materials $[2,4,6]$. The use of conductive fillers allows the production of conductive or semi-conductive polymer-based strain sensors and their resistance change when a mechanical solicitation is applied. Their sensibility is based on the percolation network of the conductive filler that suffers significant changes with the applied mechanical solicitation.

Carbonaceous materials (e.g. CNT's and graphene) are the conductive filler of choice to incorporate in a soft polymer matrix to produce a strain sensing element, especially due to the affinity between the carbon present in the filler and the carbon present in the backbone of the polymer chain $[4,7,8]$. Few and monolayered graphene was used as a strain sensing device however their stiffness and low stretchability of $6 \%$ limited their performance[5, 9]. Graphene stretchability can be improved by transferring the conductive layer to a polymeric buckled surface, however, its stretchability is limited to $20-30 \%$ [9, 10]. The graphene used in the previous works is produced by chemical vapor deposition (CVD), an expensive technique that is unsuitable for practical application. To extend the application and stretchability of graphene sensors, different processing methodologies were developed. Scaffaro et al. [11] prepared piezoresistive sensors by adding reduced graphene oxide (rGO) to the biodegradable poly(lactic acid) (PLA)-Poly (ethylene-glycol) (PEG) blends. It was reported an enhancement of the mechanical stiffness with the incorporation of the nanofiller, and their sensor was able to monitor 
submicrometric displacements with accurate sensibility. Nevertheless, the developed sensors only had a linear region up to $13 \%$.

Yan et al. [5] fabricated a strain sensor based on crumped graphene and nanocellulose. To produce flexible and stretchable sensors, they used a vacuum filtration method and encapsulate the developed conductive layer within a stretchable and flexible poly(dimethylsiloxane) (PDMS) matrix. Their sensors present a maximum stretchability of $100 \%$. Nevertheless, the samples gauge factor changes with the amount of strain applied, from 2 for small strains, up to 7 for a maximum strain of $100 \%$. Other fabrication methods were used to produce strain sensors such as printing technology [12-14], transferring and micro-molding methods [15, 16], coating techniques [1,3], liquid phase mixing [17, 18] and chemical synthesis [2, 4, 19].

The polymer matrix influences the maximum stretchability that the sensor can achieve, but also influences the sensors' overall mechanical robustness [4], and election of an appropriate polymeric matrix to achieve high-performance strain sensor is important to obtain highly stretchable sensors.

Poly(glycerol sebacate) (PGS) is a biocompatible elastomer, obtained by the polycondensation of glycerol and sebacic acid, which are low-cost raw materials, and approved for medical applications by US Food and Drug Administration [20]. PGS mechanical properties can be tuned by playing with the curing temperature and/or time. It was reported that Young's modulus of the elastomeric PGS increases with the curing temperature and/or time, due to an increase of the polymer cross-linking density [21].

The detection of a broad range of mechanical deformations requires that the sensing element can maintain a conductive path at large strains, but it should be sensitive enough to detect small strains. In this work, PGS pre-polymer was produced by a polycondensation method, and the resulting material was mixed with a graphene flake dispersion. The effect of the conductive filler concentration on the nanocomposites morphological, thermal, physical and chemical properties were systematically assessed. The graphene flakes added to the pre-polymer, followed by curing step, increased the material cross-linking density and polymer entanglement, leading to an increase of the materials Young's modulus. The electromechanical performance of the developed nanocomposites where assessed by studying the effect of the stroke speed and maximum strain applied to the sensor and their sensibility was characterized. Moreover, to demonstrate the applicability of our strain sensors as a skin-mountable device, human skin motion detection was 
performed by mounting the sensors on the surface of a finger. The developed sensors demonstrate to be capable to monitor the bending movement of the finger with good sensibility, and fast response.

\section{Experimental}

\subsection{Materials and processing}

Poly(glycerol sebacate) (PGS) was prepared according to the method explained elsewhere [20, 22]. Equimolar of glycerol (Sigma-Aldrich) and sebacic acid (Sigma-Aldrich) were mixed at $120{ }^{\circ} \mathrm{C}$ under argon atmosphere for $24 \mathrm{~h}$ to prepare a pre-polymer for further treatment. The prepolymer was poured into a Teflon mold and cured at $120^{\circ} \mathrm{C}$ for $48 \mathrm{~h}$ under a $100 \mathrm{mTorr}$ vacuum pressure. Nanocomposite films were prepared by dispersing the desired amount of graphene powder (XFNANO, TEM image is presented in S1) in tetrahydrofuran (THF, Sigma-Aldrich) solution with the help of an ultrasound bath (Bandelin, Sonorex Super RK106) for $4 \mathrm{~h}$. After dispersion, PGS pre-polymer was added to the solution and stirred at room temperature until complete dissolution. The ratio of pre-polymer to THF was $1 \mathrm{~g}$ to $5.5 \mathrm{~mL}$, respectively. The amount of graphene flakes in the solution was selected to result in a final concentration ranging from 0 to $10 \mathrm{wt} \%$ of filler presented in the PGS matrix. After complete dissolution, the solution was poured into a clean Teflon mold, and solvent evaporation was performed overnight at room temperature. Highly flexible films were obtained by curing the pre-polymer in the conditions stated above (vacuum oven at $100 \mathrm{mTorr}$, and $120^{\circ} \mathrm{C}$, for $48 \mathrm{~h}$ ).

\subsection{Nanocomposites characterization}

The graphene-PGS (fG-PGS) samples were fractured after immersion into liquid nitrogen for 30 min. The platinum coatings were deposited on the cross-section of the samples by a Dynavac Sputter Coater with the thickness of around $10 \mathrm{~nm}$. After $24 \mathrm{~h}$ in the desiccator, the images of the cross-section were taken by JEOL 7500 field emission scanning electron microscope (SEM). Raman spectra were obtained with a Horiba Jobin Yvon LabRAM HR 800 Raman spectrometer using excitation of He-Ne $632.8 \mathrm{~nm}$ with a power $13.5 \mathrm{~mW}$. Thermal gravimetric analysis (TGA) was carried out using Perkin-Elmer TGA 4000. Samples of around $10 \mathrm{mg}$ were prepared and heated from room temperature to $600{ }^{\circ} \mathrm{C}$ at $20{ }^{\circ} \mathrm{C} / \mathrm{min}$. Differential scanning calorimetry (DSC) 
experiments were conducted with a DSC 214 Polyma, from Netzsch. Samples of around $5 \mathrm{mg}$ were placed inside $40 \mu \mathrm{L}$ aluminum pans, and heated between -40 and $40{ }^{\circ} \mathrm{C}$, at a heating rate of $10{ }^{\circ} \mathrm{C} / \mathrm{min}$. All experiments (TGA and DSC) were performed under a nitrogen atmosphere. The mechanical properties of the nanocomposite samples were measured in the tensile mode, with a universal testing machine (Shimadzu EZ-L mechanical tester) fitted with a $10 \mathrm{~N}$ load cell. The prepared films with an average thickness of $1 \mathrm{~mm}$, were cut in a dog bone shape with $2 \mathrm{~mm}$ wide and $15 \mathrm{~mm}$ for the gauge length. Quasi-static measurements were performed at $1 \mathrm{~mm} / \mathrm{min}$, at room temperature $\left(\sim 22{ }^{\circ} \mathrm{C}\right)$. Mechanical cyclic experiments were performed in the same experimental conditions as quasi-static test, until a maximum strain of 5, 10 and $20 \%$, for 10 cycles, at a stroke speed of $1 \mathrm{~mm} / \mathrm{min}$.

For swelling experiments, the samples were immersed in THF and weight every day (A\&D HR200 laboratory balance) until reaching a constant weight: this was normally achieved after $48 \mathrm{~h}$ immersed in the solvent. Excessive solvent on surfaces of the sample was removed with a tissue paper, followed by a new weighting procedure. After the swollen samples were placed in a desiccator, under vacuum, during $48 \mathrm{~h}$ at $30{ }^{\circ} \mathrm{C}$ to evaporate all the solvent, followed by weighting the dry samples and the final weight was compared to the initial one. The degree of swelling was calculated as follows:

$$
\text { Swelling }(\%)=\frac{\left(W_{s}-W_{d}\right)}{W_{d}} \times 100
$$

where $W_{s}$ is the swollen mass and $W_{d}$ is the initial dry weight. The values presented are the average of three independent measurements.

\subsection{Electromechanical characterization}

Electromechanical experiments were performed by measuring the electrical resistance through metallic contacts placed on the surface of the sample, with an Agilent 34410A multimeter during the mechanical solicitation of the sample, following the method described elsewhere [20, 23]. The mechanical testing was measured with a universal testing machine (Shimadzu EZ-L mechanical tester) fitted with a $10 \mathrm{~N}$ load cell. The samples were placed between the clamps and 
submitted to a tensile cyclic load, at different stroke speeds (20 up to $50 \mathrm{~mm} / \mathrm{min}$ ), and a maximum strain between 10 and 50\%.

\section{Results and discussion}

\subsection{Sample synthesis and morphology}

The fG-PGS nanocomposite elastomers were obtained by a two step-process, the PGS prepolymer was firstly synthetised by a polycondensation method (figure 1).

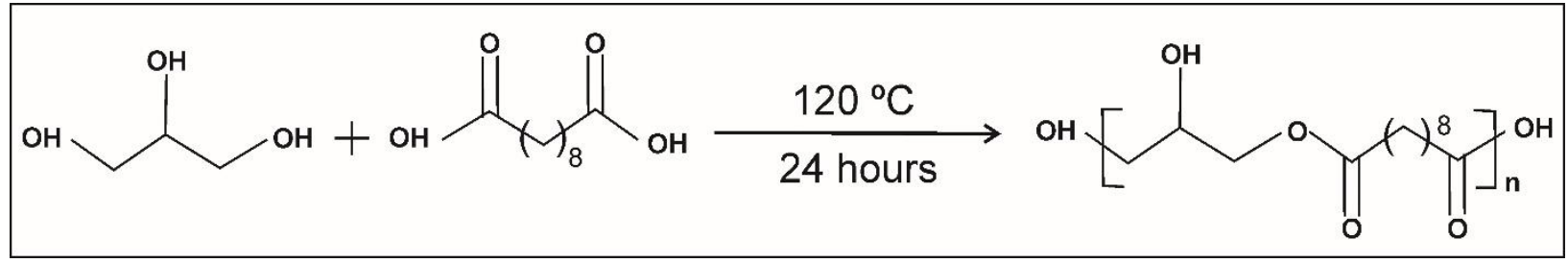

Fig.1. Polycondensation reaction of glycerol and sebacic acid to synthesize the PGS pre-polymer.

After, the PGS prepolymer was dissolved in THF, and graphene flakes were added to the solution, followed by ultrasonication, the resulting solution was found to be stable with no phase separation or segregation. Before the second processing step, the solvent could evaporate overnight at room temperature, and afterward, the sample was placed in a vacuum oven at $120{ }^{\circ} \mathrm{C}$, at $100 \mathrm{mTorr}$, for $48 \mathrm{~h}$, to obtain a cross-linked and highly flexible nanocomposite. The morphology of the cross-section of the cryo-fractured samples was analyzed by scanning electron microscopy (figure 2) and indicates that the graphene flakes were randomly dispersed among the polymer matrix, suggesting that polymer chains could separate the different graphene flakes, preventing agglomeration [24]. Furthermore, all nanocomposite samples show high flexibility and can sustain bending and twist (Fig. 2). 

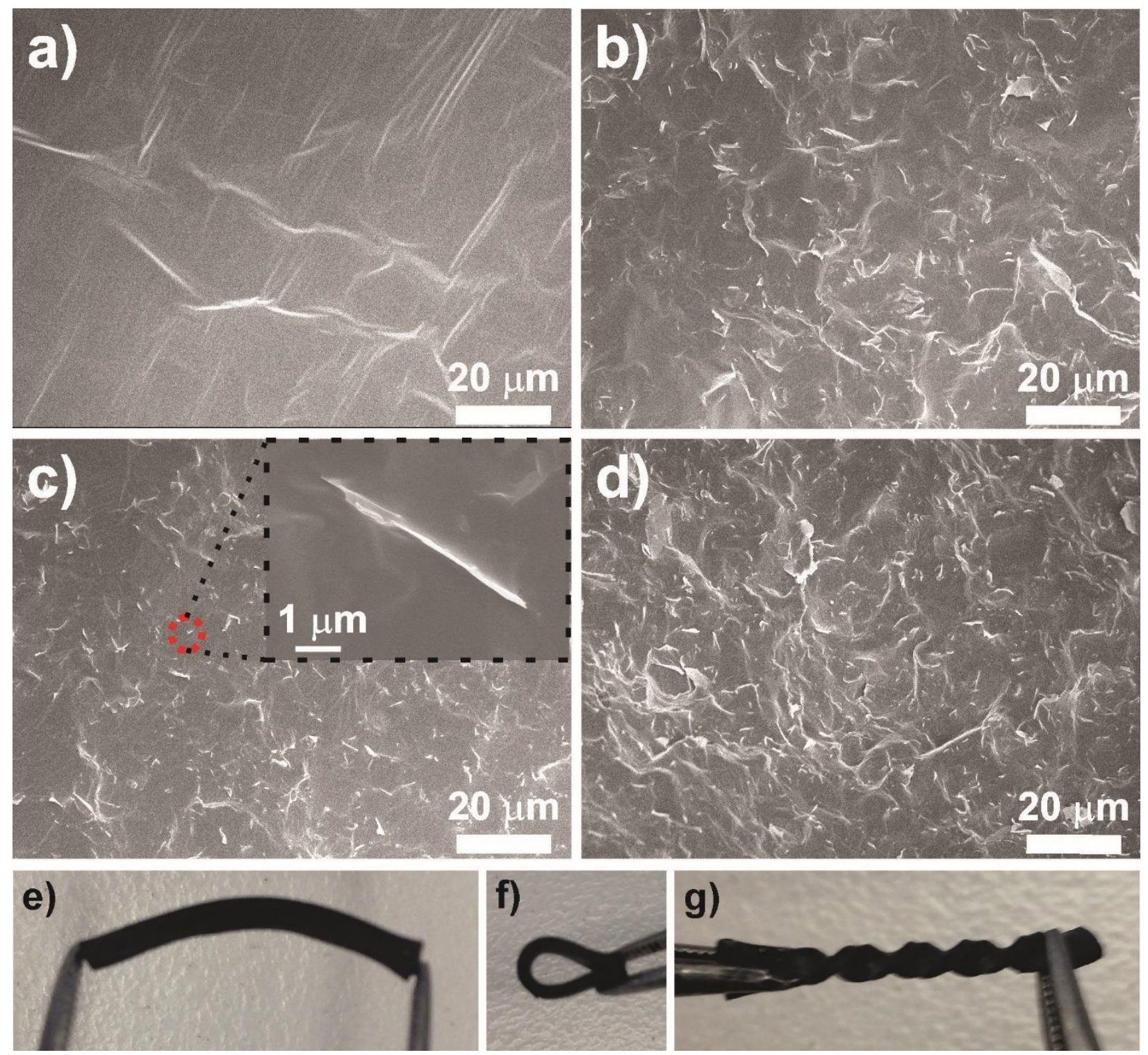

Fig. 2. Morphology of the fG-PGS elastomeric nanocomposite samples with different amounts of filler: a) pristine PGS, b) $3 \mathrm{wt} \% \mathrm{fG}$, c) $5 \mathrm{wt} \% \mathrm{fG}$, d) $10 \mathrm{wt} \% \mathrm{fG}$, e) slightly bent, f) fully bent and g) twisted sample. The bending and twisting were performed with the PGS sample with 10wt\% fG filler.

\subsection{Nanocomposite chemical and thermal properties}

The Raman spectra of the fG-PGS elastomeric nanocomposites were collected to assess the interactions between the graphene flakes and the PGS after the cross-linking procedure (figure 3). 
The Raman spectra of the graphene flakes present the characteristic absorption of the G-band at $1580 \mathrm{~cm}^{-1}$ [25], that derives from the graphite-like tangential mode. Furthermore, PGS present absorption bands at 1364 and $1450 \mathrm{~cm}^{-1}$, characteristic of the $\mathrm{CH}_{3}$ stretching [26]. After, crosslinking, the fG-PGS samples presented an increase of the graphene G-band intensity, and a new absorption band was observed at $1360 \mathrm{~cm}^{-1}$, assigned to the D-band, which corresponds to the impurities or defects present in the graphene flakes [25, 27], probably promoted by the ultrasonication procedure, but also could have a contribution from the chemical conjugation between the graphene and the PGS polymer chains, suggesting that a covalent bond between the filler and the matrix could exist (figure 3). Moreover, it was observed that the peak related to the D-band broadened with the increase of the graphene flakes incorporated into the PGS solution before cross-linking, which suggests the formation of covalent bonds between the PGS polymer chains and the graphene flakes. Chen et al. [28] observed that the D-band peak of carbon nanotubes broadened during in-situ polymerization of poly(methyl methacrylate) (PMMA), and attributed this phenomenon to covalent bonding between the PMMA chains and the CNT's.
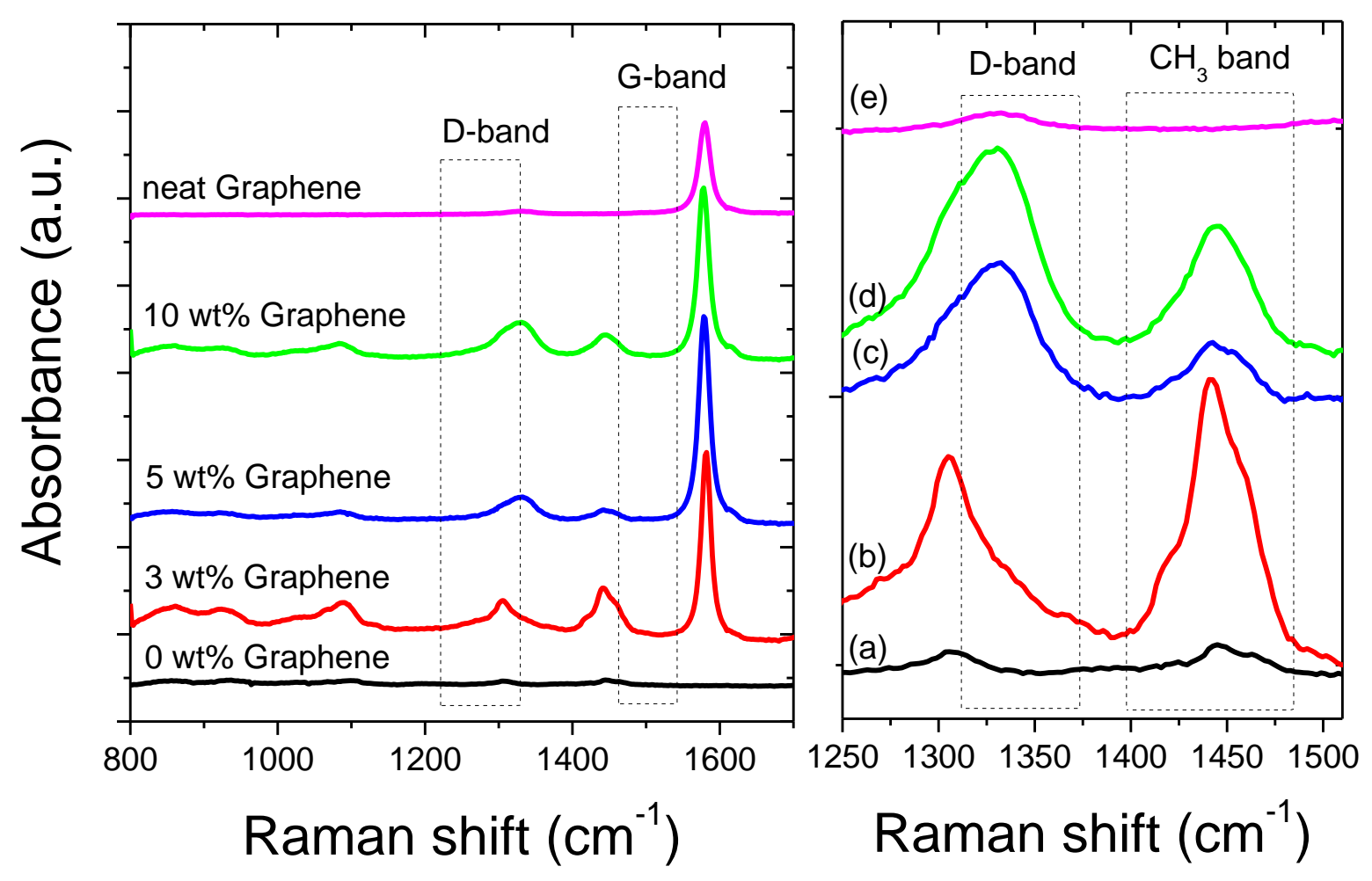

Fig. 3. Raman spectra for the PGS and fG-PGS elastomeric nanocomposite samples. 
The influence of the graphene flakes in the elastomeric matrix thermal properties was characterized by thermogravimetric analysis (TGA, figure 4a). It was observed that the incorporation of the filler does not change significantly the thermal stability of the PGS matrix, and the onset of the degradation $\left(414^{\circ} \mathrm{C}\right)$, defined by extending the pre-degradation portion of the curve to the point of the interception with a line drawn as a tangent to the steepest portion of the mass curve occurring during degradation, does not suffer significant modifications (figure 4a). Furthermore, the derivative curve of the thermal decomposition shows a single step degradation profile. Finally, the sample residual weight was calculated at $550{ }^{\circ} \mathrm{C}$, and it shows that the PGS had a residual weight of $2.3 \%$ and increases with the amount of the graphene flakes added to the elastomeric matrix, up to $12.5 \%$, showing that the initial concentration of the filler added to the solution was successfully loaded to the polymer matrix.
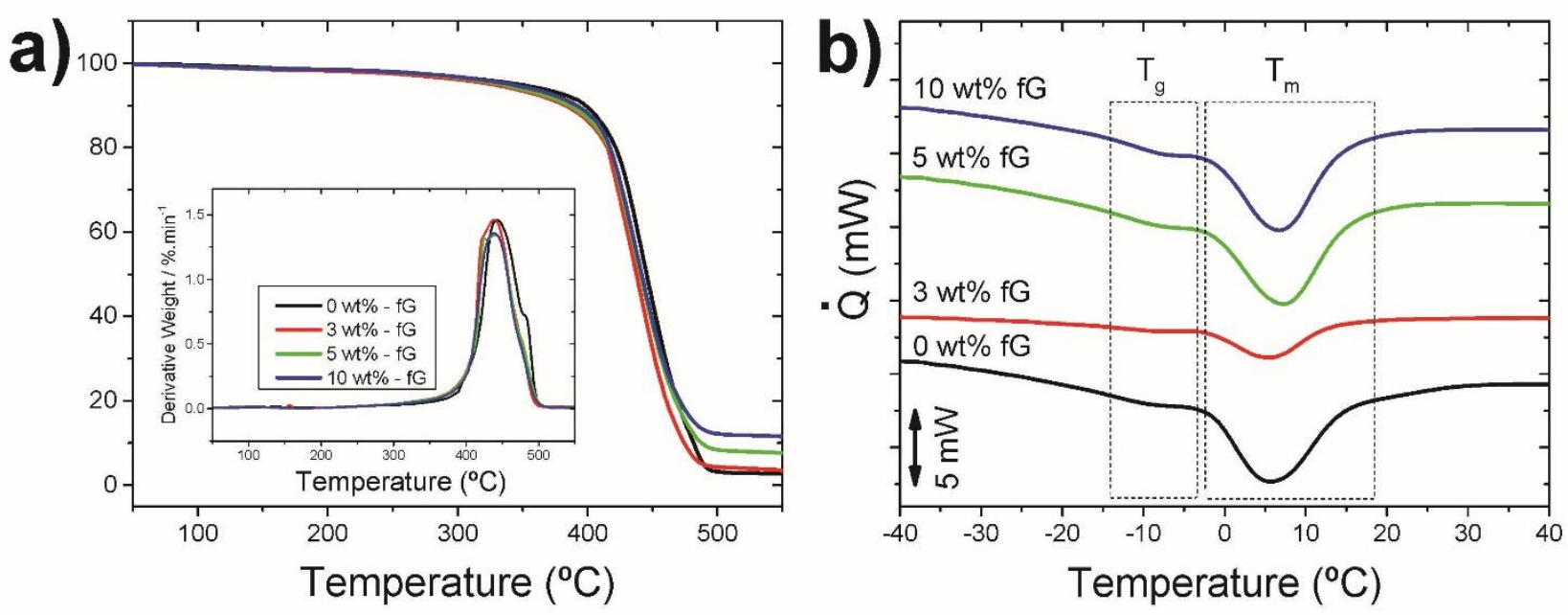

Fig. 4. a) Thermogravimetric profile, and b) DSC plots of the of the PGS and fG-PGS elastomeric nanocomposite samples.

Differential scanning calorimetry (DSC) performed on the pristine and nanocomposite PGS samples showed that the polymer had a glass transition around $-10{ }^{\circ} \mathrm{C}$, followed by a melting transition with maxima at $\sim 8{ }^{\circ} \mathrm{C}$ (figure $4 \mathrm{~b}$ ). The two thermal transition temperatures are adjacent and similar to that previously reported in the literature $[29,30]$. The enthalpy of the of the melting transition for the PGS matrix was found to be $22 \pm 2 \mathrm{~J} / \mathrm{g}$ and did not suffer significant changes with the incorporation of the filler content. Moreover, the DSC profile showed that the 
PGS is a semicrystalline polymer at temperatures below $\sim 8{ }^{\circ} \mathrm{C}$, and completely amorphous at room temperature $[21,30]$.

\subsection{Mechanical properties}

The mechanical properties of the synthesized PGS nanocomposites where investigated through quasi-static measurements (figure 5). The nanocomposite samples showed a similar trend of the stress-strain curves, consistent with the elastomeric state. Furthermore, the ultimate tensile strength (UTS) and Young's modulus $(E)$ of the synthetized samples increased with the amount of filler added to the elastomeric matrix, while the strain at break $\left(\varepsilon_{\text {break }}\right)$ decreases, as summarized in table 1 .
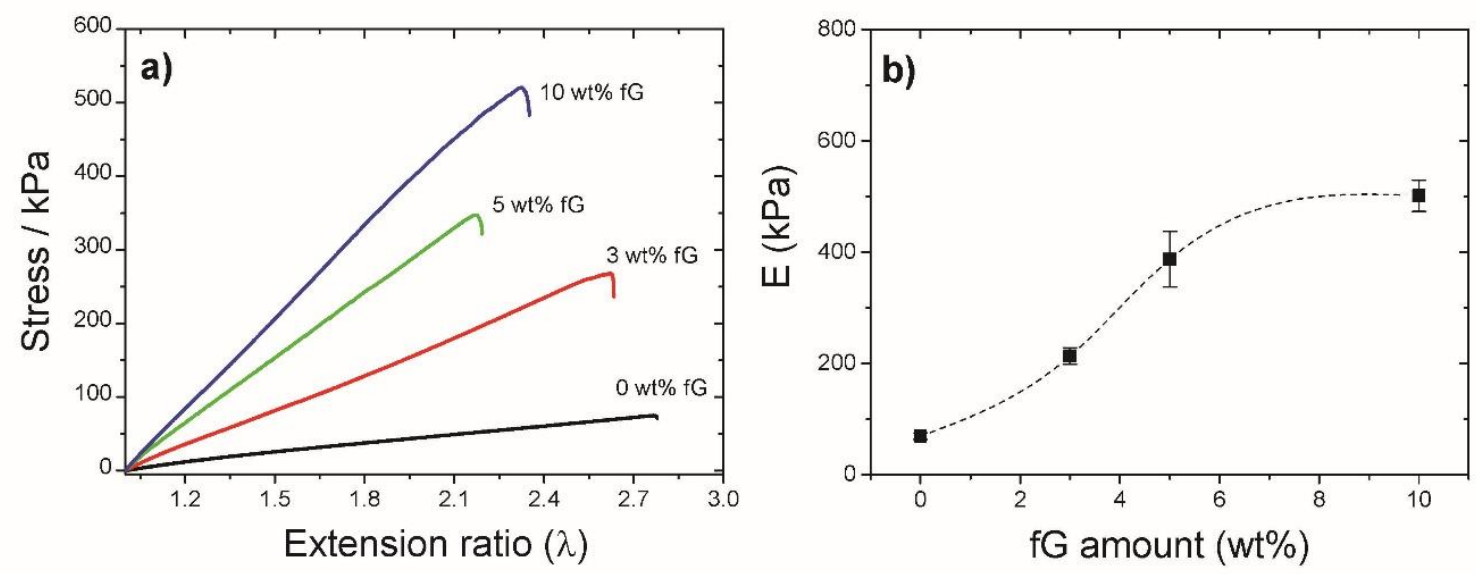

Fig. 5. a) Characteristic quasi-static mechanical, and b) evolution of Young's modulus, recorded for the fG-PGS elastomeric nanocomposite samples.

Due to the elastomeric mechanical behavior of the PGS nanocomposite samples, their mechanical stress-strain behavior can be described by the rubber elasticity theory:

$$
\sigma=\frac{E}{3}\left(\lambda-\frac{1}{\lambda^{2}}\right)
$$

where $\sigma$ is the stress, $\lambda=1+\varepsilon$ is the extension ratio, $\varepsilon$ is the tensile strain. The Young's modulus can be linearized with an $R^{2}=0.98$, for an $\lambda=2$ (table 1). PGS elastomer present a Young's modulus of $69 \pm 6 \mathrm{kPa}$, and a seven-fold increase was observed with the incorporation 
of $10 \mathrm{wt} \%$ of graphene flakes, and this increase could be attributed to the strong interactions between oxygen-containing groups (e.g., hydroxyls and carbonyl groups) of the graphene flakes and the hydroxyl groups of the PGS polymer chains (figure 1), that restricts the polymer chain movement during the mechanical solicitation. As a result, a more effective load transfer across the filler and the polymer chains interface is obtained, resulting in a significant increase of the nanocomposites UTS. The strong interaction between the filler and the matrix, added to the high aspect ratio of the graphene flakes, reduces the polymer chain mobility, leading to a decrease in the deformation at break ( $\varepsilon_{\text {break }}$, table 1). Another factor that contribute for this behaviour is the presence of aggregates among the soft matrix (figure 2), especially for the samples with higher amount of filler. Potts et al. [8] found that the dispersion plays an important role in the mechanical behaviour of reduced graphene-natural rubber nanocomposites. In their work, they found that the rGO agglomerates prevents interparticle diffusion of polymer chains, providing cohesion between the latex parts of the natural rubber, increasing the nanocomposite UTS, but leading to a reduction in the $\varepsilon_{\text {break }}$.

Our results showed a remarkable enhancement in mechanical properties when compared to the ones found in the literature. When the graphene flakes were added to the PMMA, an increase of $80 \%$ and $20 \%$ in Young's modulus and UTS, respectively was reported by Ramanthan et al. [31]. Similar behavior was reported for functionalized graphene oxide sheets dispersed in a poly(vinyl alcohol) (PVA) matrix [32]. Graphene oxide-poly(imide) composite films were prepared by insitu polymerization by Wang et al. [33]. In their work, they showed that increasing the amount of graphene oxide (GO) into the polymerization reaction of poly(imide) (PI) led to an 8-fold increase of the UTS and Young's modulus of the nanocomposite samples. Furthermore, a decrease in the GO-PI samples tensile strain was attributed to the strong interfacial interactions that restricted the polymer chain movement. Our results demonstrate that the incorporation of graphene flakes into the prepolymer, followed by cross-linking leads to the formation of a hybrid polymer nanocomposite elastomeric material with enhanced mechanical performance when compared to reinforced materials. 
Table 1. Mechanical properties and cross-linking density of PGS and composites with different fG amounts.

\begin{tabular}{cccc}
\hline $\begin{array}{c}\text { Graphene Flakes } \\
\mathbf{w t} \%\end{array}$ & $\begin{array}{c}\boldsymbol{\varepsilon}_{\text {break }} \\
\boldsymbol{\%}\end{array}$ & $\begin{array}{c}\boldsymbol{U T S} \\
\mathbf{k P a}\end{array}$ & $\begin{array}{c}\mathbf{n} \\
\mathbf{m o l} / \mathbf{m}^{\mathbf{3}}\end{array}$ \\
\hline 0 & $174 \pm 21$ & $67 \pm 8$ & $6.4 \pm 0.8$ \\
3 & $150 \pm 12$ & $271 \pm 18$ & $28.9 \pm 2.0$ \\
5 & $114 \pm 16$ & $342 \pm 23$ & $52.6 \pm 6.8$ \\
10 & $136 \pm 29$ & $488 \pm 54$ & $68.1 \pm 3.8$ \\
\hline
\end{tabular}

The effect of the mechanical energy absorbed by the polymer network was evaluated through cyclic tensile measurements for different strain loadings (5, 10 and $20 \%$ deformation, figure 6). Pure PGS showed an almost perfect elastomeric behavior with low mechanical energy loss, even for tensile strains up to $20 \%$ (figure 6a). Moreover, the incorporation of the graphene flakes into the PGS network enhances the amount of energy absorbed without compromising the elastic behavior of the nanocomposites (figure 6b). This behavior is probably due to the strong interaction between the graphene flakes and the PGS polymer backbone that results in improved load transfer efficiency within the cross-linking network (figure $6 \mathrm{c}$ and d).

The cross-linking density $(n)$ of the synthetised nanocomposites can be calculated according to:

$$
E=3 R T n
$$

Where $T$ is the absolute temperature, and $R$ is the universal gas constant. The evolution of the cross-linking density with the amount of graphene flakes added to the PGS elastomeric matrix is presented in table 1.

In equation 3, $n$ represents the number of active network chain segments per unit of volume that contributes for the overall mechanical response of the elastomeric nanocomposite samples, and it may be due to chemical cross-links and/or physical chain entanglements [34].

An increase of the cross-linking density of more than four-fold was observed, and a value of 6.4 \pm 0.8 and $68.1 \pm 3.8 \mathrm{~mol} / \mathrm{m}^{3}$ was calculated for the pristine and for the sample with $10 \mathrm{wt} \% \mathrm{fG}$, suggesting that the filler contributes not only to the physical entanglement, but also chemical cross-linking of the polymeric chains. 

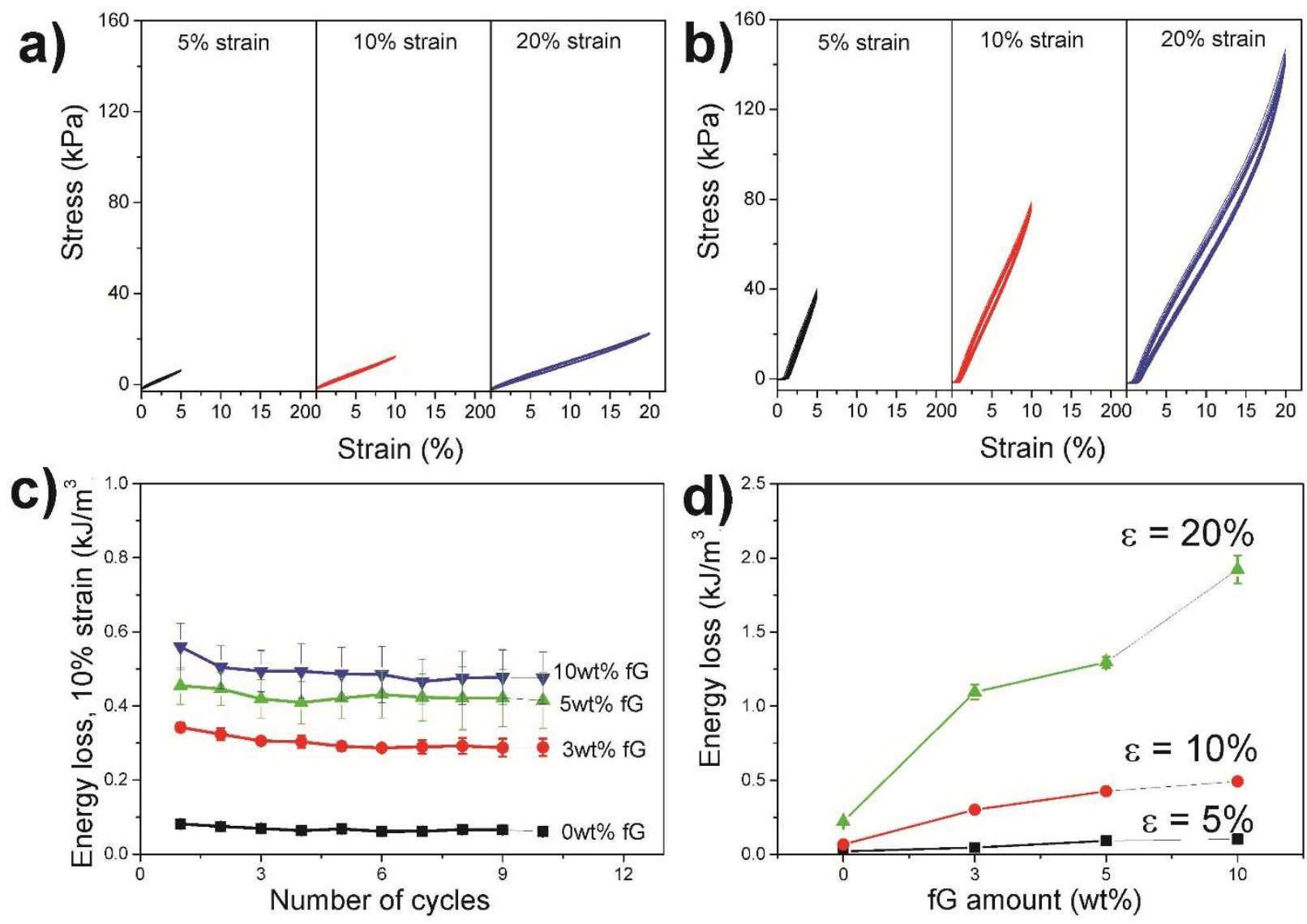

Fig. 6. Cyclic mechanical loading-unloading data with a maximum strain of 5, 10 and 20\%: a) pristine PGS, b)fG-PGS with $10 \mathrm{wt} \%$ of filler, c) energy loss recorded for different cycles for the fG-PGS nanocomposite samples, recorded at a maximum strain of $10 \%$ and d) evolution of the energy loss of the fG-PGS samples, recorded for the different maximum strains applied.

\subsection{Swelling behavior and crosslinking density}

The cross-linking density of an elastomeric material can also be estimated through swelling experiments. Figure 7 shows the macroscopic morphology of the PGS and fG-PGS nanocomposite samples, before and after being swelled to equilibrium in THF solution. The amount of solvent that the sample could uptake was calculated according to equation 1, and the values are presented in table 2. 


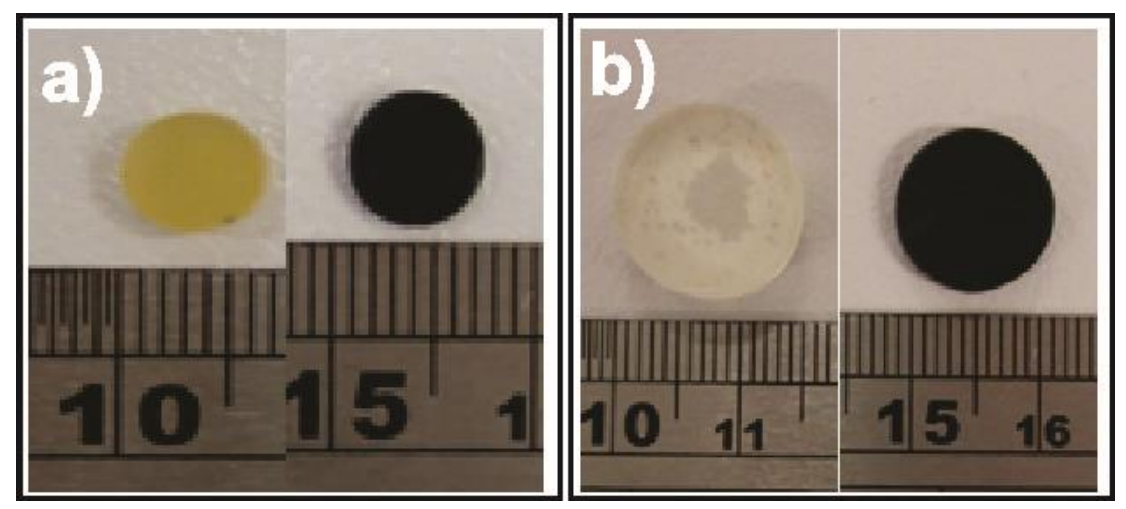

Fig. 7. Macroscopic morphology of the PGS and fG-PGS (10 wt $\%$ ) samples: a) dry sample and b) after swelling the THF solvent until equilibrium.

The cross-linking density $(n)$ of the PGS and fG-PGS elastomeric nanocomposites can be calculated through the solvent swelling. According to Flory [35], the density of a network polymer chain segments is given by:

$$
\mathrm{n}=\frac{1}{2} \frac{\left[\ln \left(1-v_{p}\right)+v_{p}+\chi v_{p}^{2}\right]}{v_{s}\left(v_{p} / 2-v_{p}{ }^{1 / 3}\right)}
$$

where $v_{s}$ and $v_{p}$ are the volume fraction of solvent and polymer in the equilibrium swollen network, respectively, and $\chi$ is Flory-Huggins polymer-solvent interaction parameter [35]. For a tetra-functional affine network, the cross-linking density of a swollen material can be calculated through the Flory - Rehner theory:

$$
v_{p}=\left[1+\left(\frac{m_{e q}-m_{d}}{m_{d}}\right)\left(\frac{\rho_{p}}{\rho_{s}}\right)\right]^{-1}
$$

where, $\rho_{s}$ and $\rho_{p}$ are the solvent and polymer density, respectively.

The Flory-Huggins PGS solvent interaction parameter assumes a constant value for a specific pair solvent-polymer and does not suffer changes with the cross-linking density of the polymer chains [36]. A value of $\chi=0.43$ was reported for the THF-PGS polymer [37], and it was used to solve equation 5 and obtain the cross-linking density of the PGS samples with different loadings of fG. The cross-linking density of the synthesized fG-PGS elastomeric samples increases with 
the amount of filler added to the pre-polymer before cross-linking (table 2). Moreover, the crosslinking density calculated through the rubber elasticity theory (table 1) and through the swelling experiments (table 2) for pristine PGS is similar, which corroborates that a value of 0.43 could be used for the Flory-Huggins polymer-solvent interaction parameter.

If one assumes that the solvent interaction parameter is a constant for a specific pair polymersolvent [36], and the only experimental parameter changed was the amount of filler added to the PGS matrix before the curing of the elastomeric matrix, the obtained results for the cross-linking density measured through the swelling experiments suggests that the fG filler is covalently bonded to the PGS polymer chains, increasing its cross-linking density, and is in agreement with the Raman spectroscopy and mechanical results (Fig. 3 and table 1). Additionally, the differences between observed in the cross-linking density calculated by the rubber elasticity theory (table 1) and through the Flory mathematical model, suggests that the incorporation of graphene flakes increases the polymer chemical cross-linking, but it also promotes enhanced physical entanglement of the macromolecular chains [36]. The values obtained for the cross-linking degree of the PGS are in accordance with the ones reported in the literature, where $n$ values between 5 and $20 \mathrm{~mol} / \mathrm{m}^{3}$ where obtained for samples prepared in similar conditions as the ones of this work [37-39].

Table 2. Crosslinking density of different samples using the swelling method and mechanical method.

\begin{tabular}{ccc}
\hline Graphene Flakes & Swelling degree & $\boldsymbol{n}$ \\
wt \% & $(\%)$ & $\left(\chi=\mathbf{0 . 4 3}, \mathbf{~ m o l} / \mathbf{m}^{\mathbf{3}}\right)$ \\
\hline 0 & $1141 \pm 55$ & $8.0 \pm 0.6$ \\
5 & $767 \pm 4$ & $17.0 \pm 0.8$ \\
10 & $614 \pm 13$ & $23.0 \pm 0.6$ \\
\hline
\end{tabular}




\subsection{Electromechanical performance}

To characterize the performance of the developed nanocomposites as piezoresistive sensors, the samples were clamped in a universal testing machine, and the mechanical performance was recorded at the same time as the samples electrical resistance variation (figure 8a). A nanocomposite is considered as a highly stretchable strain sensor if their electrical resistance can follow linearly the mechanical solicitation for deformations higher than $20 \%$ [4], and this is only possible if the conductive paths are not permanently destroyed during the mechanical solicitation [40].

In the present work, a mechanical tensile strain up to 50\% was applied to the samples to access the robustness and the linearity of the material electrical performance with the mechanical solicitation. It was observed that the nanocomposite samples change in resistance showed excellent linearity $\left(R^{2}=0.98, \mathrm{~S} 2\right)$ for a wide range of tensile deformations applied, from 0 to $50 \%$ (figure 8a), which define the developed nanocomposites as highly stretchable strain sensors [4]. When the mechanical strain was applied, an increase of the resistance was observed (figure 8a), and this behavior is due to the increase in distance between the conductive fillers.

The sensibility of the sensors can be obtained through the calculation of the gauge factor (GF), defined as the electrical resistance variation $\left(\frac{\Delta R}{R_{0}}\right)$ related to the applied mechanical tensile strain $\varepsilon$ :

$$
G F=\frac{\frac{\Delta R}{R_{0}}}{\varepsilon}
$$

The GF was calculated for the samples submitted to different stroke speeds (figure $8 \mathrm{~b}$ ) and tensile strains applied (figure 8c). The developed fG-PGS sensors showed that their sensibility is independent of the stroke speed and/or tensile strain applied (figure $8 \mathrm{~b}$ and c). To this date, only metallic strain gauges exhibit this highly desirable feature. Having stretchable strain sensors containing this characteristic could replace the current commercial metallic strain gauges, that have a limit of stretchability with less than $5 \%$, for more reliable and highly stretchable elastomeric sensors.

Stretchable strain sensors present a diverse range of GF values, depending on matrix properties, conductive fillers used piezoresistive mechanisms and micro/nanostructures of the strain sensors 
[4]. Jeong et al. [41] prepared strain sensors from fragmentized graphene foam, and they found that the GF values changes from 2.4 for an applied tensile strain of $8.5 \%$ to 15 , for the same sensor, stretched in same conditions but with a final strain of $77 \%$. They suggested that at higher strains, the decreasing of the percolation network becomes more crucial with fewer conductive paths. GF values up to $10^{6}$ were reported for graphene woven fabrics deposited on the surface of a PDMS substrate. Nevertheless, these giant GF values are limited to small strains $(\varepsilon<10 \%)$, and with limited linearity [42]. Other work, reports that the nano-graphene coated natural rubber, presents GF values <1 for an applied tensile strain of $100 \%$, and the values found was due to the lower initial resistance of the sensors, promoted by a dense conductive network formation on the top of the rubber substrate [43].

The piezoresistive sensors developed in this work showed greater sensibility when compared to commercial strain gauges made from either metal alloys or semiconductors. Moreover, from the mechanical property point of view, the fG-PGS devices can sustain with a linear response, much higher strain than other sensing materials [4].

The developed sensor can be shaped and attached to any complex configuration, e.g. the human finger. A strip of fG-PGS was cut and attached to a finger to detect its motion. Owing to its soft nature and high stretchability (figure 5) the sensor could follow the movement of the finger without constraining its motion (figure 8d). Finally, the resistance decreased up to $80 \%$ of its initial value when the bending angle rise from 0 (extended finger) to $90^{\circ}$ (fully closed finger). The negative value of the change in resistance is probably due to the decrease in the sensor thickness promoted by the bending movement of the finger. These results in an increase in the amount of contact points between the graphene flakes, leading to an overall increase of the percolation network.

Human skin has Young's modulus ranging between $200 \pm 50$ for the forearm dermis up to $250 \pm 75$ $\mathrm{kPa}$ for the palm dermis [44]. The developed strain gauges have Young's modulus that matches the skin that is highly stretchable and skin-mountable. and allied to Its mechanical performance combined with its electromechanical properties and electrical sensibility, means they have potential to monitor human movement without causing discomfort or constraints to the movable components or the user. 

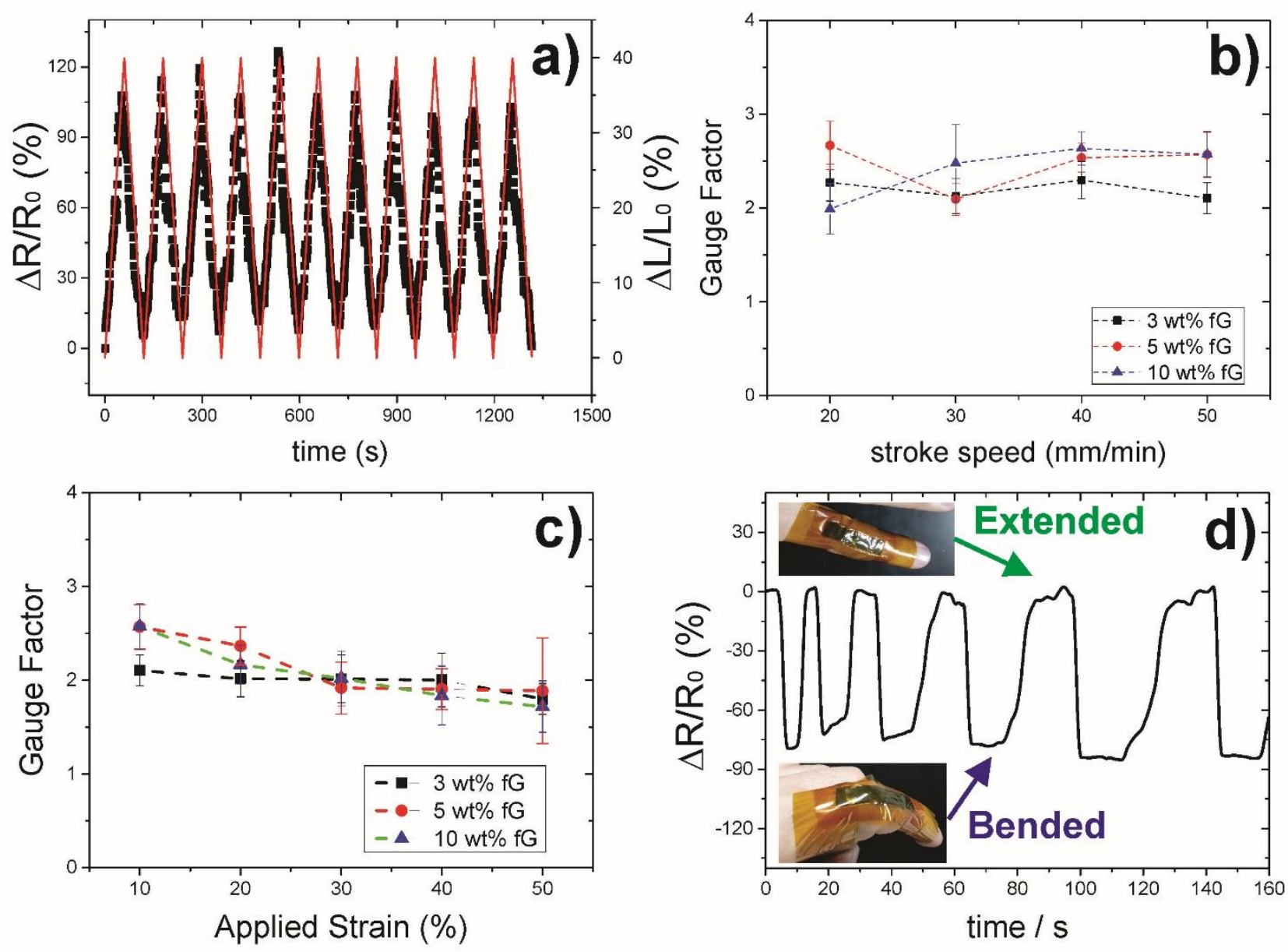

Fig. 8. a) Response of the strain sensor under repeated mechanical stretching and release cycles for the sample with $10 \mathrm{wt} \%$ fG filler, b) Evolution of the strain GF with stroke speed, c) Evolution of the GF with the applied strain, and d) Motion detection of the index finger, for the sample with $10 \mathrm{wt} \%$ fG filler.

\section{Conclusions}

Highly stretchable elastomeric nanocomposite strain sensors were developed through the synthesis of a PGS prepolymer, followed by incorporation of the conductive filler and crosslinking of the polymer chains. The developed sensors showed almost perfect elastomeric behavior. It was observed that the incorporation of the filler enhanced the mechanical properties, without compromising its elastomeric behavior and the thermal properties of the polymeric matrix. Furthermore, it was observed that the incorporation of the graphene flakes led to an 
increase of the cross-linking density of the polymer, contributing to the overall increase of Young's modulus from $69 \pm 6$ for the pristine PGS up to $501 \pm 28 \mathrm{kPa}$ for the sample with $10 \mathrm{wt} \%$ filler. The cross-linking density of the nanocomposite samples, calculated through the mechanical rubber elasticity theory is almost double the value calculated through the FloryHuggins model, suggesting the filler actively promotes chemical cross-linking sites for the polymer chains to attach, and increases the physical entanglement of the polymer chains. It was found that the sensibility of the synthesized piezoresistive sensors is independent of the stroke speed or applied strain, and a GF of $\sim 2$ was calculated for all tested samples. Furthermore, the developed sensors showed a remarkable capability to monitor the bending movement of the finger. Finally, the performance revealed by the synthesized piezoresistive sensors will contribute to the next generation of highly stretchable smart materials for sensing and control human motion with minimum discomfort.

\section{Acknowledgments}

The authors acknowledge financial support from China Scholarship Council (CSC), UPA from University of Wollongong and Australian Research Council (ARC) for Future Fellowship (Z.Y. Jiang). The authors acknowledge use of facilities within the UOW Electron Microscopy Centre and the Australian National Nanofabrication Facility - Materials node for equipment use. The authors acknowledge Mr. Jiangshan Zhang and Dr. Tony Romeo for SEM imaging.

\section{References:}

[1] J.J. Park, W.J. Hyun, S.C. Mun, Y.T. Park, O.O. Park, Highly Stretchable and Wearable Graphene Strain Sensors with Controllable Sensitivity for Human Motion Monitoring, ACS Applied Materials \& Interfaces 7(11) (2015) 6317-6324.

[2] T. Yamada, Y. Hayamizu, Y. Yamamoto, Y. Yomogida, A. Izadi-Najafabadi, D.N. Futaba, K. Hata, A stretchable carbon nanotube strain sensor for human-motion detection, Nat Nano 6(5) (2011) 296-301.

[3] V. Sencadas, R. Mutlu, G. Alici, Large area and ultra-thin compliant strain sensors for prosthetic devices, Sensors and Actuators A: Physical 266(Supplement C) (2017) 56-64.

[4] M. Amjadi, K.-U. Kyung, I. Park, M. Sitti, Stretchable, Skin-Mountable, and Wearable Strain Sensors and Their Potential Applications: A Review, Advanced Functional Materials 26(11) (2016) 1678-1698. 
[5] C. Yan, J. Wang, W. Kang, M. Cui, X. Wang, C.Y. Foo, K.J. Chee, P.S. Lee, Highly Stretchable Piezoresistive Graphene-Nanocellulose Nanopaper for Strain Sensors, Advanced Materials 26(13) (2014) 2022-2027.

[6] J.D. Pegan, J. Zhang, M. Chu, T. Nguyen, S.-J. Park, A. Paul, J. Kim, M. Bachman, M. Khine, Skin-mountable stretch sensor for wearable health monitoring, Nanoscale 8(39) (2016) 17295-17303.

[7] J.H. Kang, C. Park, J.A. Scholl, A.H. Brazin, N.M. Holloway, J.W. High, S.E. Lowther, J.S. Harrison, Piezoresistive characteristics of single wall carbon nanotube/polyimide nanocomposites, Journal of Polymer Science Part B: Polymer Physics 47(10) (2009) 994-1003.

[8] J.R. Potts, D.R. Dreyer, C.W. Bielawski, R.S. Ruoff, Graphene-based polymer nanocomposites, Polymer 52(1) (2011) 5-25.

[9] K.S. Kim, Y. Zhao, H. Jang, S.Y. Lee, J.M. Kim, K.S. Kim, J.-H. Ahn, P. Kim, J.-Y. Choi, B.H. Hong, Large-scale pattern growth of graphene films for stretchable transparent electrodes, Nature 457(7230) (2009) 706-710.

[10] Y. Wang, R. Yang, Z. Shi, L. Zhang, D. Shi, E. Wang, G. Zhang, Super-Elastic Graphene Ripples for Flexible Strain Sensors, ACS Nano 5(5) (2011) 3645-3650.

[11] R. Scaffaro, A. Maio, G. Lo Re, A. Parisi, A. Busacca, Advanced piezoresistive sensor achieved by amphiphilic nanointerfaces of graphene oxide and biodegradable polymer blends, Composites Science and Technology 156 (2018) 166-176.

[12] S. Yao, Y. Zhu, Wearable multifunctional sensors using printed stretchable conductors made of silver nanowires, Nanoscale 6(4) (2014) 2345-2352.

[13] J.T. Muth, D.M. Vogt, R.L. Truby, Y. Mengüç, D.B. Kolesky, R.J. Wood, J.A. Lewis, Embedded 3D Printing of Strain Sensors within Highly Stretchable Elastomers, Advanced Materials 26(36) (2014) 6307-6312.

[14] B.F. Gonçalves, J. Oliveira, P. Costa, V. Correia, P. Martins, G. Botelho, S. LancerosMendez, Development of water-based printable piezoresistive sensors for large strain applications, Composites Part B: Engineering 112 (2017) 344-352.

[15] C.-X. Liu, J.-W. Choi, Analyzing resistance response of embedded PDMS and carbon nanotubes composite under tensile strain, Microelectronic Engineering 117(Supplement C) (2014) $1-7$.

[16] D.J. Cohen, D. Mitra, K. Peterson, M.M. Maharbiz, A Highly Elastic, Capacitive Strain Gauge Based on Percolating Nanotube Networks, Nano Letters 12(4) (2012) 1821-1825.

[17] H. Deng, M. Ji, D. Yan, S. Fu, L. Duan, M. Zhang, Q. Fu, Towards tunable resistivity-strain behavior through construction of oriented and selectively distributed conductive networks in conductive polymer composites, Journal of Materials Chemistry A 2(26) (2014) 10048-10058.

[18] J.-H. Kong, N.-S. Jang, S.-H. Kim, J.-M. Kim, Simple and rapid micropatterning of conductive carbon composites and its application to elastic strain sensors, Carbon 77(Supplement C) (2014) 199-207.

[19] J. Zhao, C. He, R. Yang, Z. Shi, M. Cheng, W. Yang, G. Xie, D. Wang, D. Shi, G. Zhang, Ultra-sensitive strain sensors based on piezoresistive nanographene films, Applied Physics Letters 101(6) (2012) 063112.

[20] Y. Yan, V. Sencadas, J. Zhang, G. Zu, D. Wei, Z. Jiang, Processing, characterisation and electromechanical behaviour of elastomeric multiwall carbon nanotubes-poly (glycerol sebacate) nanocomposites for piezoresistive sensors applications, Composites Science and Technology 142(Supplement C) (2017) 163-170. 
[21] R. Rai, M. Tallawi, A. Grigore, A.R. Boccaccini, Synthesis, properties and biomedical applications of poly(glycerol sebacate) (PGS): A review, Progress in Polymer Science 37(8) (2012) 1051-1078.

[22] Y. Wang, G.A. Ameer, B.J. Sheppard, R. Langer, A tough biodegradable elastomer, Nature biotechnology 20(6) (2002) 602-6.

[23] P. Costa, A. Ferreira, V. Sencadas, J.C. Viana, S. Lanceros-Méndez, Electro-mechanical properties of triblock copolymer styrene-butadiene-styrene/carbon nanotube composites for large deformation sensor applications, Sensors and Actuators A: Physical 201 (2013) 458-467.

[24] S. Stankovich, D.A. Dikin, G.H.B. Dommett, K.M. Kohlhaas, E.J. Zimney, E.A. Stach, R.D. Piner, S.T. Nguyen, R.S. Ruoff, Graphene-based composite materials, Nature 442(7100) (2006) 282-286.

[25] D.G. Papageorgiou, I.A. Kinloch, R.J. Young, Graphene/elastomer nanocomposites, Carbon 95(Supplement C) (2015) 460-484.

[26] R. Rai, M. Tallawi, J.A. Roether, R. Detsch, N. Barbani, E. Rosellini, J. Kaschta, D.W. Schubert, A.R. Boccaccini, Sterilization effects on the physical properties and cytotoxicity of poly(glycerol sebacate), Materials Letters 105 (2013) 32-35.

[27] Y. Shi, D. Xiong, J. Li, N. Wang, The water-locking and cross-linking effects of graphene oxide on the load-bearing capacity of poly(vinyl alcohol) hydrogel, RSC Advances 6(86) (2016) 82467-82477.

[28] B. Chen, M. Cinke, J. Li, M. Meyyappan, Z. Chi, J.P. Harmon, P.A. O'Rourke Muisener, L. Clayton, J. D'Angelo, Modifying the Electronic Character of Single-Walled Carbon Nanotubes Through Anisotropic Polymer Interaction: A Raman Study, Advanced Functional Materials 15(7) (2005) 1183-1187.

[29] A.K. Gaharwar, A. Patel, A. Dolatshahi-Pirouz, H. Zhang, K. Rangarajan, G. Iviglia, S.-R. Shin, M.A. Hussain, A. Khademhosseini, Elastomeric nanocomposite scaffolds made from poly(glycerol sebacate) chemically crosslinked with carbon nanotubes, Biomaterials Science (2015).

[30] W. Cai, L. Liu, Shape-memory effect of poly (glycerol-sebacate) elastomer, Materials Letters 62(14) (2008) 2171-2173.

[31] RamanathanT, A.A. Abdala, StankovichS, D.A. Dikin, M. Herrera Alonso, R.D. Piner, D.H. Adamson, H.C. Schniepp, ChenX, R.S. Ruoff, S.T. Nguyen, I.A. Aksay, R.K. Prud'Homme, L.C. Brinson, Functionalized graphene sheets for polymer nanocomposites, Nat Nano 3(6) (2008) 327-331.

[32] J. Liang, Y. Huang, L. Zhang, Y. Wang, Y. Ma, T. Guo, Y. Chen, Molecular-Level Dispersion of Graphene into Poly(vinyl alcohol) and Effective Reinforcement of their Nanocomposites, Advanced Functional Materials 19(14) (2009) 2297-2302.

[33] J.-Y. Wang, S.-Y. Yang, Y.-L. Huang, H.-W. Tien, W.-K. Chin, C.-C.M. Ma, Preparation and properties of graphene oxide/polyimide composite films with low dielectric constant and ultrahigh strength via in situpolymerization, Journal of Materials Chemistry 21(35) (2011) 13569-13575.

[34] L.H. Sperling, Introduction to physical polymer science, Wiley, New York, 1992.

[35] P.J. Flory, Principles of polymer chemistry, Ithaca : Cornell Univ. Pr.1953.

[36] S.P. Rout, G.B. Butler, Crosslinking of diene polymers via 'ENE' reaction with bistriazolinediones, Polymer Bulletin 2(8) (1980) 513-520. 
[37] Q.Z. Chen, A. Bismarck, U. Hansen, S. Junaid, M.Q. Tran, S.E. Harding, N.N. Ali, A.R. Boccaccini, Characterisation of a soft elastomer poly(glycerol sebacate) designed to match the mechanical properties of myocardial tissue, Biomaterials 29(1) (2008) 47-57.

[38] Y. Li, W.D. Cook, C. Moorhoff, W.-C. Huang, Q.-Z. Chen, Synthesis, characterization and properties of biocompatible poly(glycerol sebacate) pre-polymer and gel, Polymer International 62(4) (2013) 534-547.

[39] X. Li, A.T.L. Hong, N. Naskar, H.-J. Chung, Criteria for Quick and Consistent Synthesis of Poly(glycerol sebacate) for Tailored Mechanical Properties, Biomacromolecules 16(5) (2015) 1525-1533.

[40] J.C. Dawson, C.J. Adkins, Conduction mechanisms in carbon-loaded composites, Journal of Physics: Condensed Matter 8(43) (1996) 8321.

[41] Y.R. Jeong, H. Park, S.W. Jin, S.Y. Hong, S.-S. Lee, J.S. Ha, Highly Stretchable and Sensitive Strain Sensors Using Fragmentized Graphene Foam, Advanced Functional Materials 25(27) (2015) 4228-4236.

[42] X. Li, R. Zhang, W. Yu, K. Wang, J. Wei, D. Wu, A. Cao, Z. Li, Y. Cheng, Q. Zheng, R.S. Ruoff, H. Zhu, Stretchable and highly sensitive graphene-on-polymer strain sensors, Scientific Reports 2 (2012) 870.

[43] S. Tadakaluru, T. Kumpika, E. Kantarak, W. Sroila, A. Panthawan, P. Sanmuangmoon, W. Thongsuwan, P. Singjai, Highly stretchable and sensitive strain sensors using nano-graphene coated natural rubber, Plastics, Rubber and Composites 46(7) (2017) 301-305.

[44] C. Li, G. Guan, R. Reif, Z. Huang, R.K. Wang, Determining elastic properties of skin by measuring surface waves from an impulse mechanical stimulus using phase-sensitive optical coherence tomography, Journal of The Royal Society Interface 9(70) (2012) 831-841. 


\section{Supplementary Information}

$$
\text { Yi Yan }{ }^{1+} \text {, Michael Potts }{ }^{1+} \text {, Zhengyi Jiang }{ }^{1, *} \text {, Vitor Sencadas }{ }^{1,2 *}
$$

${ }^{1}$ School of Mechanical, Materials, Mechatronic and Biomedical Engineering, University of Wollongong, Wollongong, NSW 2522, Australia

${ }^{2}$ ARC - Center of Excellence for Electromaterials Science, University of Wollongong, 2522 NSW, Australia

+ both authors contribute equally

*Corresponding author e-mail address: victors@uow.edu.au; jiang@uow.edu.au

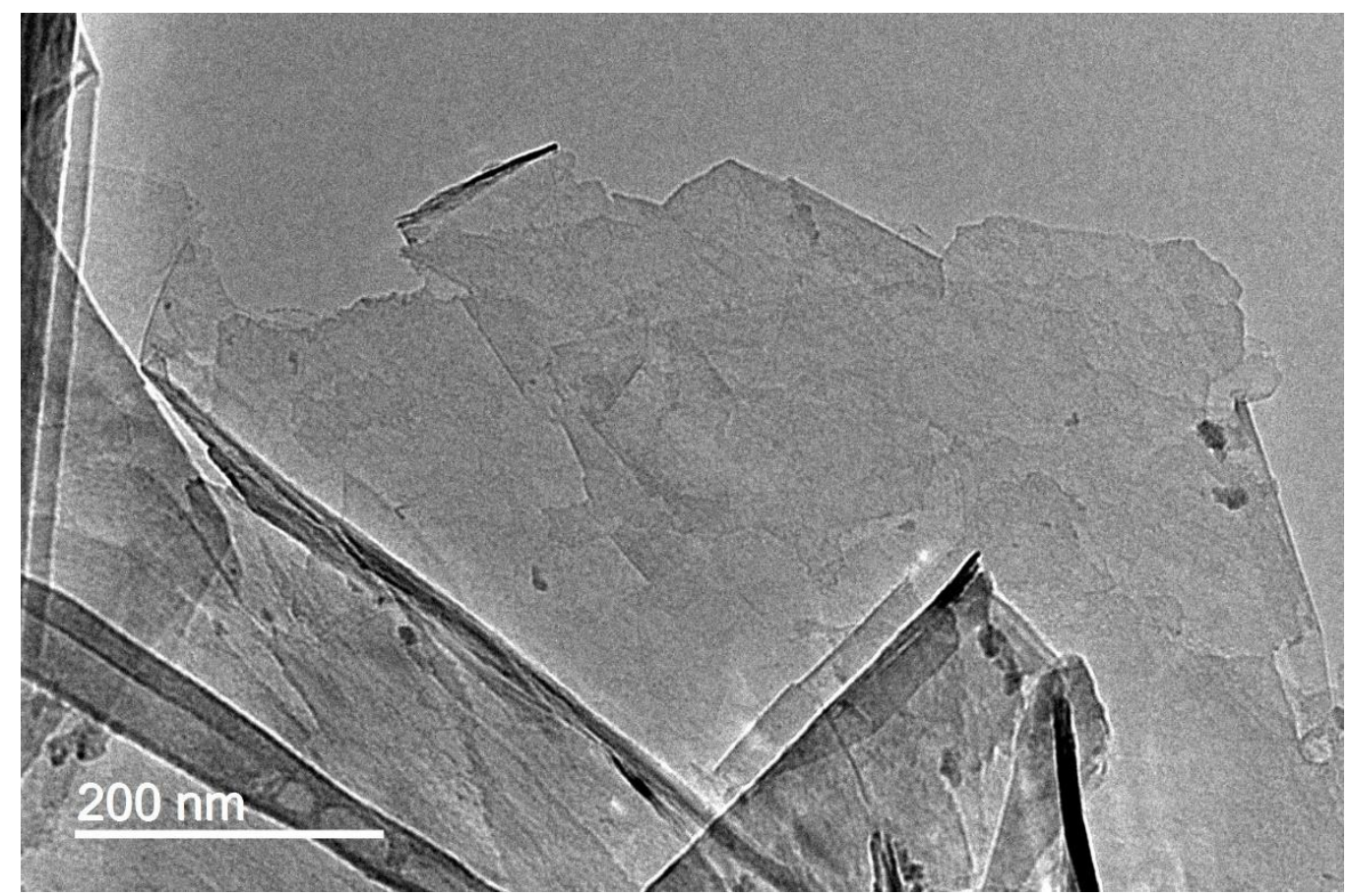

S1. Transmission electron microscopy image for the Graphene flake used to produce the fG-PGS nanocomposites. 


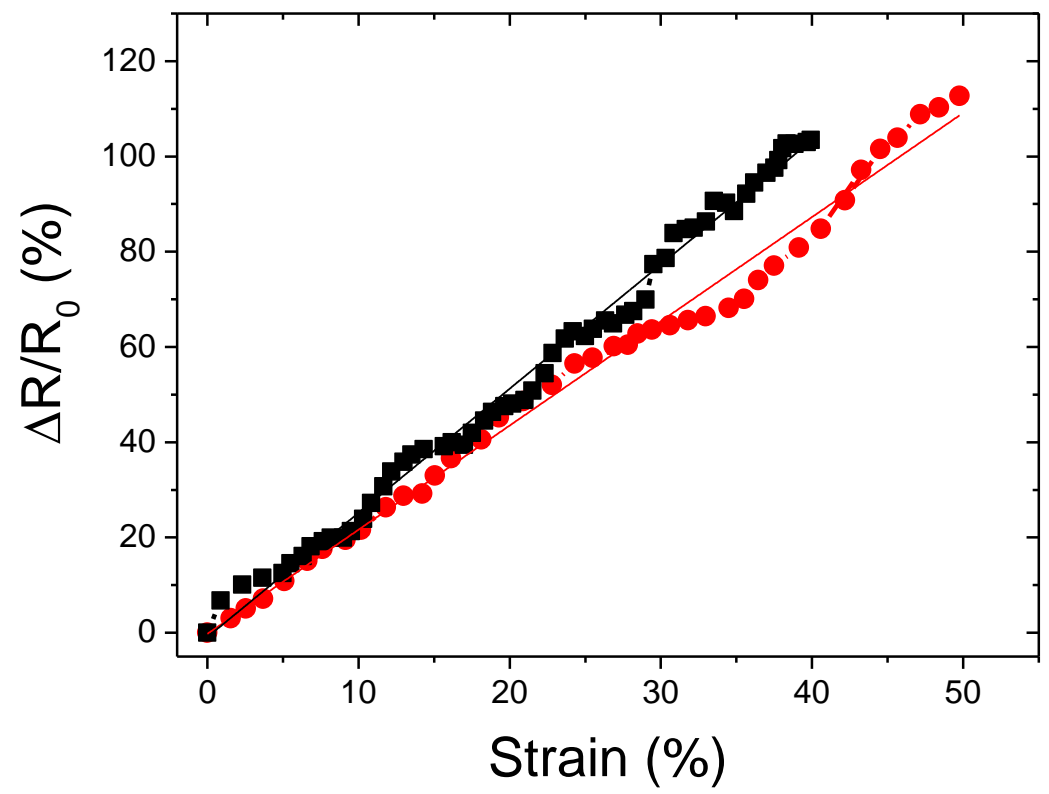

S2. Representative linear fitting (line) used to evaluate the GF for the fG-PGS nanocomposite sample with $10 \mathrm{wt} \%$ filler, under $40 \%$ and $50 \%$ deformation.

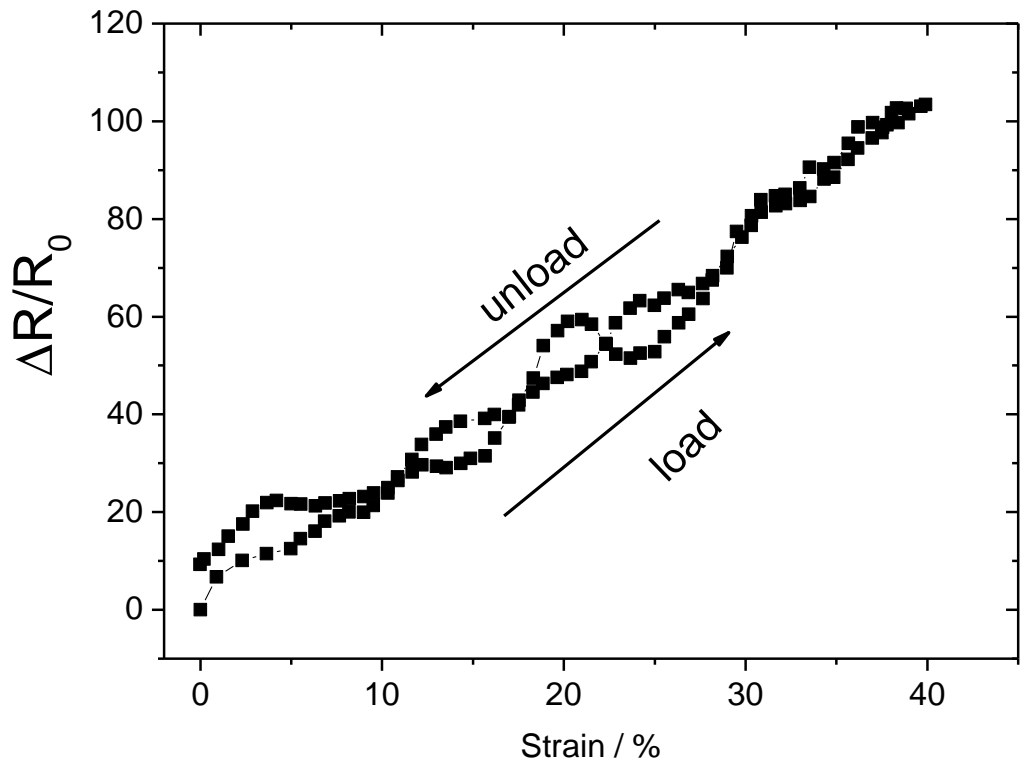

S3. Electric hysteresis loop recorded during the $10^{\text {th }}$ electromechanical cycle for the $f G-P G S$ nanocomposite sample with $10 \mathrm{wt} \%$ filler. 